\title{
Potential for jaktinib hydrochloride to treat cytokine storms in patients with COVID-19
}

\author{
Xianmin Meng, Yun Ling ${ }^{2}$, Li Zhang ${ }^{1}$, Qian Zhang ${ }^{1}$, Ping Dong ${ }^{1}$, Tongyu Zhu ${ }^{3}$, \\ Hongzhou $\mathrm{Lu}^{4, *}$
}

${ }^{1}$ Department of Pharmacy, Shanghai Public Health Clinical Center, Fudan University, Shanghai, China;

${ }^{2}$ Department of Infection, Shanghai Public Health Clinical Center, Fudan University, Shanghai, China;

${ }^{3}$ Department of Urology, Shanghai Public Health Clinical Center, Fudan University, Shanghai, China;

${ }^{4}$ Department of Infection and Imuunity, Shanghai Public Health Clinical Center, Fudan University, Shanghai, China.

\begin{abstract}
SUMMARY Severe acute respiratory syndrome coronavirus 2 (SARS-CoV-2) infection has become a serious public health threat to the whole world, and the number of infected is still rising dramatically at this moment. Several studies have confirmed that cytokine storms play a critical role in causing a case to worsen from mild to severe or critical. The current treatment for cytokine storms is limited, so the international medical community is focusing on a specific and effective remedy. Jaktinib hydrochloride is a broad spectrum JAK inhibitor. It can inhibit cytokine-induced immune activation by multiple mechanisms and also slow viral proliferation by inhibiting AAK1 without causing unacceptable toxicity. Jaktinib hydrochloride has potential for the treatment of patients with coronavirus disease 2019 (COVID-19).
\end{abstract}

Keywords severe acute respiratory syndrome coronavirus 2 (SARS-CoV-2), coronavirus disease 2019 (COVID-19), cytokines, jaktinib hydrochloride, feasibility

\section{Introduction}

The outbreak of novel coronavirus-related pneumonia (coronavirus disease 2019, or COVID-19) not only poses a serious threat to Chinese public health and economic and social development but has also become a global public health problem. Among all of the challenges for medical professionals, treatment of critical patients has always been the top priority (1-4). Studies indicate that a "cytokine storm" is an important signal that a patient's condition had changed from mild to severe or critical and even life-threatening. In fact, how to suppress that cytokine storm is one of the keys to hopefully curing those critical patients (5-7).

In the "Guidelines for the treatment of COVID-19, Version 7" issued by Chinese health authorities, an elevated level of human interleukin-6 (IL- 6) should be treated with tocilizumab in patients in relatively severe condition with extensive lung lesions $(8,9)$. Suppressing cytokine storms prior to their occurrence or in their early stages has been crucial to reducing the severity of COVID-19 in patients and improving their prognosis. This implies that a medication to suppress cytokine storms would hold promise. Jaktinib hydrochloride is a broad spectrum novel JAK (Janus kinase, JAK) inhibitor that is expected to inhibit cytokine storms in patients with COVID-19 $(10,11)$.

The current article describes the pathogenesis of and relevant treatments for cytokine storms induced by severe acute respiratory syndrome coronavirus 2 (SARSCoV-2) and how jaktinib hydrochloride may play a role in inhibiting those storms in clinical practice.

\section{Pathogenesis of SARS-CoV-2 infection}

SARS-CoV-2 belongs to a new type of coronavirus of the genus Betacoronavirus. It has an envelope, and the particles are round or oval, often polymorphic, with a diameter of $60-140 \mathrm{~nm}$. SARS-CoV-2 and SRAS-CoV have similar pathogenic mechanisms and pathological processes. SARS-CoV-2 invades cells with S protein by receptor-mediated endocytosis of angiotensin-converting enzyme 2 (ACE2) on pulmonary alveolar II epithelial cells (AT2) $(12,13)$. After entering the lung cells, the virus replicates in large quantities, triggering humoral and cellular immune responses, perhaps systematically. In the early stages of infection, the body deploys a large number of $\mathrm{T}$ cells to fight the virus while some patients will have a lower cellular immunity because of over consumption of T cells. Meanwhile, anti-virus- 
specific antibodies will also begin to be produced. As the disease progresses, the destruction of lung cells by the virus increases, and the body's immune response and leukocyte-related cytokine release further kills and eliminates the virus along with necrotic lung cells. In addition, the excessive increase in inflammatory factors, such as various interleukins, can trigger a series of cytokine storms. These excessive inflammatory reactions backfire, resulting in injury and causing overwhelming pulmonary inflammation or secondary fibrotic lesions; they can also ultimately lead to respiratory failure or even death in severe cases.

At present, one of the direct clinical manifestations of COVID-19 in severe cases is the multiple organ damage caused by cytokine storms. Although the virus is the initiating factor, immune overexpression caused by cytokine storms is a direct cause of systematic injury. For instance, clinical evidence indicates that after reaching the turning point of cytokine storms, highflow oxygen and invasive ventilation are ineffective, eventually resulting in the use of extracorporeal membrane oxygenation (ECMO) (5). One of the direct reasons for this is that patients with COVID-19 have scarce surfactants in the alveoli, impaired lung interstitia, impaired ventilation, and thusly as a consequence of unconvertible ventilation failure, ECMO is the only potential rescue technique. Although the factors that cause COVID-19 to worsen from mild to severe or critical are similar, the main problem has to be handled in a quite different manner. The major problem in a mild case is the viral infection, while the main problem in severe or exacerbated illness is the series of cytokine storms.

\section{SARS-CoV-2-induced cytokine storm}

Cytokine storm syndrome (CSS) is usually defined as abnormal activation of the immune system and uncontrolled release of cytokines, leading to systemic inflammation and multiple organ dysfunction. Several studies have indicated that CSS is also the main reason for death due to SARS and MERS (14-17). Cytokine storms can directly damage the pulmonary capillary mucosa, leading to alveolar edema and inactivation of surfactant proteins, which can further induce inflammatory factors to diffuse in the lungs, cause alveolar structures to damaged and degenerate, and result in pulmonary ventilation dysfunction (18). During a cytokine storm, the immune system produces proinflammatory cytokines that facilitate the progression of the disease while anti-inflammatory factors can accelerate pathogen removal and contribute to tissue repair. The extent of the immune response is correlated with prognosis to an extent.

As mentioned, the first step in the early stages of infection is a non-specific immune response; as the infection progresses, $\mathrm{T}$ or $\mathrm{B}$ lymphocytes begin to function as the main effector cells of specific immune responses (14). In the stage of a specific immune response, pulmonary endothelial cells produce a large volume of inflammatory factors (19). Tumor necrosis factor (TNF), IL-1, IL-8, and monocyte chemoattractant protein 1 (MCP-1) are the first to increase, followed by IL-6 and IL-10 (20). IL-6 is produced by stimulation caused by TNF and IL-1 $\beta$, and the peripheral blood concentration can be used to evaluate the intensity of the systemic inflammatory response (21).

The mechanism by which SARS-CoV-2 causes cytokine storms is not fully understood. Early studies indicated that patients with COVID-19 have high levels of expression of IL-1B, IFN- $\gamma$, interferon-induced protein 10 (IP-10), and MCP-1, and these may activate a Th1 cell response (22). In comparison to patients with mild or moderate COVID-19 not necessitating treatment in the ICU, patients with severe COVID-19 in the ICU have higher serum levels of granulocyte colony-stimulating factor (GCSF), IP-10, MCP-1, and macrophage inflammatory protein $1 \mathrm{~A}$ (MIP-1A) as well as higher levels of expression of TNF- $\alpha$, suggesting a correlation between the severity of disease and the occurrence of a cytokine storm. In addition, serum levels of IL-2, IL-7, and IL-10 are also significantly elevated (22). An autopsy of patients with COVID-19 revealed diffuse alveolar injury with fibrous mucuslike exudates in both lungs, large numbers of shed cells and the formation of transparent membranes, and inflammatory infiltration of monocytes in the alveolar stroma (23). In peripheral blood samples from patients who died of COVID-19, $\mathrm{CD}^{+}$and $\mathrm{CD}^{+} \mathrm{T}$ lymphocyte counts were decreased, human leukocyte antigen DR (HLA-DR) and the CD38 double-positive T lymphocyte ratio significantly increased, implying that these immune $\mathrm{T}$ lymphocytes are excessively activated. In $\mathrm{CD}^{+} \mathrm{T}$ lymphocytes, an incremental increase in the proportion of $\mathrm{CC}$ chemokine receptor 4 (CCR-4) and CCR6-positive highly proinflammatory Th17 cells was also detected. $\mathrm{CD}^{+} \mathrm{T}$ lymphocytes contain high concentrations of cytotoxic particles, suggesting that during the occurrence of cytokine storms in COVID-19, excessive activation of $\mathrm{T}$ lymphocytes may be accompanied by serious immune impairment (24). Based on the above findings, Janus kinase/signal transducer and activator of transcription (JAK/STAT) pathways may exist in patients with COVID-19, resulted in immune overreaction and inducing a cytokine storm, eventually causing an excessive immune inflammatory response and/or secondary lung injury/fibrosis, possibly leading to respiratory failure.

\section{Current treatment options for SARS CoV-2- induced cytokine storm}

Previous versions of the "Guidelines for the treatment of COVID-19" issued by Chinese health authorities 
have displayed a gradual increase in the recognition of cytokine storms in COVID-19, and recommended therapeutic strategies are being updated accordingly. The fifth edition mentions that a cytokine test is necessary if available for the first time, and corticosteroid treatment in an appropriate dosage that does not exceed the equivalent of methylprednisolone $1-2 \mathrm{mg} / \mathrm{kg} /$ day (25) can be administered to patients with inflammatory overactivation for a brief period (3-5 days). The seventh edition indicates that severe and critical cases usually involve complex inflammatory factors, consistently reduced peripheral blood lymphocytes, and conversely increased levels of inflammatory cytokines such as IL-6 and C-reactive protein. After the seventh edition, immunotherapy has been recommended for the management of severe and critical patients, tocilizumab is indicated for patients with severe disease, extensive lung injury, and elevated IL-6 levels according to laboratory results $(8)$. The following medications and therapies are listed to inhibit cytokine storms in patients with COVID-19:

i) Glucocorticoids. For critical cases or patients with immune activation, the benefits and risks should be promptly assessed, and caution should always be exercised regarding duration and dosage. Usually, the principle is to start with small doses $(\leq 0.5$ to $1.0 \mathrm{mg}$ / $\mathrm{kg} \cdot \mathrm{d}$ methylprednisolone or equivalent) and short-term treatment $(\leq 7 \mathrm{~d})(8,26)$.

ii) IL-6 antagonist (tocilizumab), a recombinant humanized anti- IL-6 receptor monoclonal antibody, is most frequently used in clinical practice for treatment of general infection and the ensued inflammation caused by a cytokine storm $(8,27)$. Recent studies have found tocilizumab to be an effective therapeutic strategy, and especially in patients with severe COVID-19 $(9,28)$. Therefore, if laboratory results reveal elevated IL-6 in a systematic infection, tocilizumab is indicated.

iii) Chloroquine and hydroxychloroquine. By inhibiting the production and release of TNF and IL-6, these two drugs suppress the occurrence of a cytokine storm. Nearly 300 clinical studies are currently examining different dosages of chloroquine, hydroxychloroquine, or both to treat COVID-19 worldwide, and some of them actually involve severe and critical patients. However, there has been some controversy regarding the safety and efficacy of the two drugs in the treatment of COVID-19 $(29,30)$.

iv) Vitamin $\mathrm{C}$ in high doses. Vitamin $\mathrm{C}$ is administered intravenously at a dose of 100 to $200 \mathrm{mg} / \mathrm{kg}$ daily. Some evidence has potentially indicated significant improvement in the oxygenation index (31).

v) Ulinastatin. Ulinastatin is a broad-spectrum hydrolase inhibitor, 1.6 million units every $8 \mathrm{~h}$. Under mechanical ventilation, when the oxygenation index is $>$ $300 \mathrm{mmHg}$, it can be reduced to 1 million units/d (31).

vi) Unfractionated heparin. Anticoagulation therapy protects endothelial cells and reduces cytokine release, alleviating the immune response. Unfractionated heparin (3 to $15 \mathrm{IU} / \mathrm{kg}$ per hour) is recommended when FDP $\geq$ $10 \mu \mathrm{g} / \mathrm{mL}$ and/or D-dimer $\geq 5 \mu \mathrm{g} / \mathrm{mL}$ (31).

vii) Continuous renal replacement therapy (CRRT). Inflammatory factors can be continuously removed, including cytokines and complement activation products by extracorporeal blood purification, thereby suppressing the systemic inflammatory response $(32,33)$.

\section{Jaktinib hydrochloride as a drug to treat SARS- CoV-2-induced cytokine storm?}

Jaktinib hydrochloride is a broad-spectrum JAK inhibitor with an innovative structure. It is a class 1 new drug according to the Chinese classification, and its development has been sponsored by the National Thirteenth Five-Year Plan. Currently, this compound has passed the milestone of phase I, II, and III clinical trials for five indications, including idiopathic pulmonary fibrosis and primary myelofibrosis.

5.1. Rationale to treat SARS CoV-2- induced cytokine storm by jaktinib hydrochloride

The JAK family of non-receptor protein-tyrosine kinases consists of JAK1, JAK2, JAK3, and tyrosine kinase 2 (TYK2). JAK transmits intracellular signals from cell surface receptors that act on various cytokines and growth factors involved in inflammation and immune function, thus affecting the immune process. Different receptors can activate different subtypes of JAK, thus exhibiting differentiated biological functions (34). JAK-STAT signaling pathways are associated with the pathogenesis of a variety of inflammatory diseases, such as rheumatoid arthritis (35). Over the past 30 years, JAK has become a very important target, and several JAK inhibitors such as baricitinib, ruxolitinib, and tofacitinib have been successfully developed and marketed for the treatment of primary myelofibrosis, bone marrow fibrosis, and rheumatoid arthritis $(36,37)$. As a new JAK inhibitor, jaktinib hydrochloride can suppress JAK1, JAK2, JAK3, and TYK2 at the cell level, block the JAK-STAT signaling pathways to stop the release of cytokines by multiple mechanisms including IL-2, of IL-4, of IL-6, IL-7, and IL-10, and significantly relieve the inflammation due to immune reactions.

The pathophysiological mechanism of SARS$\mathrm{CoV}$ and MERS-CoV has yet to be fully clarified. Earlier studies found that increased levels of proinflammatory factors (such as IL-1B, IL-6, IL-12, IFN- $\gamma$, IP-10, and MCP-1) in plasma of SARS patients are associated with pneumonia and severe lung injury $(38,39)$. Patients with a MERS-CoV infection were also found to have elevated levels of pro-inflammatory factors (such as IFN- $\gamma$, TNF- $\alpha$, IL-15, and IL-17) in plasma (17). Patients with SARS-CoV-2 have been 
found to have elevated levels of Th2 cytokines (e.g. IL-4 and IL-10) besides elevated levels of cytokines. This inhibits the inflammatory response and differs in a SARS-CoV infection. JAK inhibitors can inhibit the JAK-STAT signaling pathway, reducing the level of IP-10 expression induced by S protein in mouse lungs and repairing the damage caused by a virus on the immune system. JAK inhibitors do not completely inhibit the antiviral action of IFN- $\gamma$. Therefore, in addition to antiviral therapy, JAK inhibitors can provide a new therapeutic strategy for virus-mediated immune damage, and especially in the treatment of excessive immune inflammation and/or secondary lung injury or fibrosis caused by SARS-CoV-2 $(10,40)$. A prospective, multicenter, single-blind, randomized controlled phase II trial has indicated that recipients of ruxolitinib had numerically faster clinical improvement, significant improvement according to chest CT, faster recovery from lymphopenia, and less severe adverse reactions compared to a group receiving a placebo (11). By inhibiting JAK1, JAK2, JAK3, etc., jaktinib hydrochloride can prevent abnormal activation of the JAK-STAT pathway and inhibit leukocyte-mediated inflammation via multiple channels. It is expected to significantly counteract the inflammation/cytokine storm caused by the immune response in patients with COVID-19 (Figure 1). Jaktinib hydrochloride inhibits the development of pneumonia caused by cytokine storms via a similar mechanism and thus prevents the development of pulmonary fibrosis.
Studies have found that SARS-COV-2 invades cells by ACE2 receptors on AT2 alveolar epithelial cells in the lungs (12). AP2- associated protein kinase 1 (AAK1) is an endocytosis regulator. Inhibition of AAK1 may block virus transmission in cells and the intracellular assembly of virus particles. Blocking this process of infection may help to slow disease progression (41). Jaktinib hydrochloride can inhibit the activity of AAK1 and may block endocytosis whereby the virus enters a cell, interrupting the process of virus assembly, reducing the proliferation of the virus in vivo, and having direct antiviral action (Figure 1).

In animal experiments, jaktinib hydrochloride significantly inhibited inflammation and reduced tissue damage. In a bleomycin-induced pulmonary fibrosis model, jaktinib hydrochloride significantly alleviated the damage and inflammation of the terminal bronchioles and accompanying small pulmonary arteries in areas of fibrotic lesions and surrounding areas. Moreover, jaktinib hydrochloride had a significant result on lung injury and inflammation in fibrotic lesions in comparison to the positive control drug pirfenidone. A study of lung samples from autopsied patients with confirmed COVID-19 revealed characteristic interstitial lung disease, thereby indicating the fundamental potential for use of jaktinib hydrochloride.

5.2. Registered clinical trials on JAK inhibitors including jaktinib hydrochloride to treat COVID-19

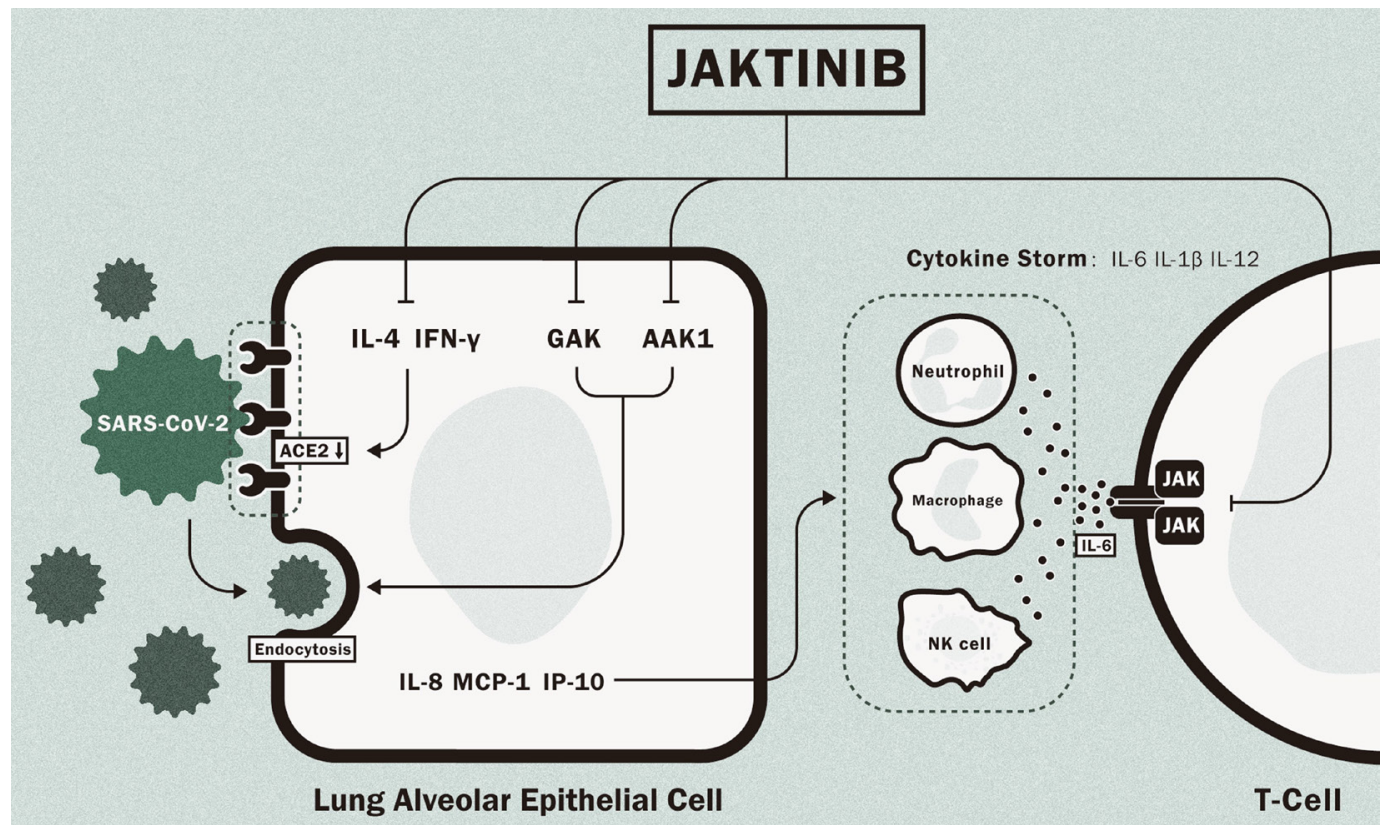

Figure 1. Mechanism of jaktinib in inhibition of viral entry and initiation of a cytokine storm. (1) Via its spike protein, SARS-CoV-2 binds to ACE2 on the surface of pulmonary alveolar II epithelial cells, inducing activation of clathrin-mediated endocytosis. AAK1- and GAK-mediated phosphorylation of clathrin adapter proteins starts the assembly of the clathrin cage around the enclosed virus. Inhibition of AAK1 and GAK by jaktinib thereby inhibits viral entry. (2) Jaktinib stops the release of cytokines such as, IL-1 $\beta$, IL-12, and IL-6, avoiding the occurrence of a cytokine storm by suppressing JAK. (SARS-CoV-2, severe acute respiratory syndrome coronavirus 2; ACE2, angiotensin-converting enzyme 2; AAK1, AP2-associated protein kinase 1; GAK, cyclin G-associated kinase; JAK, Janus kinase) 
In view of the pathogenesis of SARS-CoV-2 and the role of cytokine storms in the progression of COVID-19 in severe cases, JAK inhibitors may be an effective option for treatment of COVID-19. In light of the worldwide pandemic, JAK inhibitors are now being used clinically to treat COVID-19. To survey clinical trials on JAK inhibitors as an intervention strategy in COVID-19, a search of the databases of ClinicalTrials.gov was conducted using COVID-19, ruxolitinib, baricitinib, and tofacitinib as keywords. Prior to May 28, 2020, 28 studies related to JAK inhibitors and COVID-19 were identified (Table 1). Those studies included 24 interventional studies and 4 observational studies. The estimated study start date was mainly from March to May 2020 and the estimated completion date was mainly from May to October 2020. Thirteen studies had an estimated enrollment of 100 patients or more, 5 had an estimated enrollment of 100 to 200 , and 10 had an estimated enrollment of more than 200. Notably, there were only 4 multicenter randomized controlled trials, 3 with baricitinib and 1 with ruxolitinib.

Because many patients with severe respiratory disease due to COVID-19 have features consistent with cytokine release syndrome and increased activation of the JAK/STAT pathway, JAK inhibitors might play a useful role in treating those patients. As shown by Table 1, this view is shared by numerous researchers in many countries and regions around the world. A clinical trial on jaktinib hydrochloride by the current authors is still in the recruitment stage (Registration NO.: ChiCTR2000030170). This study will follow the principles of the Declaration of Helsinki and it has been approved by the ethics committee of the Shanghai Public Health Clinical Center.

\section{Conclusion}

Humans are now facing SARS-COV-2 globally. There is no established specific treatment available to inhibit cytokine storms in patients with COVID-19, so effective treatments should have top priority. New drugs or vaccines are not likely to be developed soon, but nevertheless the potential of existing innovative medicines could be explored as a plausible alternative (42). As a broad-spectrum JAK inhibitor, jaktinib hydrochloride can mitigate the cytokine storms by inhibiting the immune activation induced by IL-6, IL2, IL-1, granulocyte-macrophage colony-stimulating factor, IFN- $\gamma$, etc. In addition, jaktinib hydrochloride has the potential to inhibit the proliferation of SARSCoV-2 in vivo by inhibiting AAK1 activity. For patients whose condition has worsened from mild to severe or potentially life-threatening due to cytokine storms, jaktinib hydrochloride may play a dual role in inhibiting cytokine storms and virus replication. This could potentially delay or reverse disease progression, thereby reducing the mortality of COVID-19.

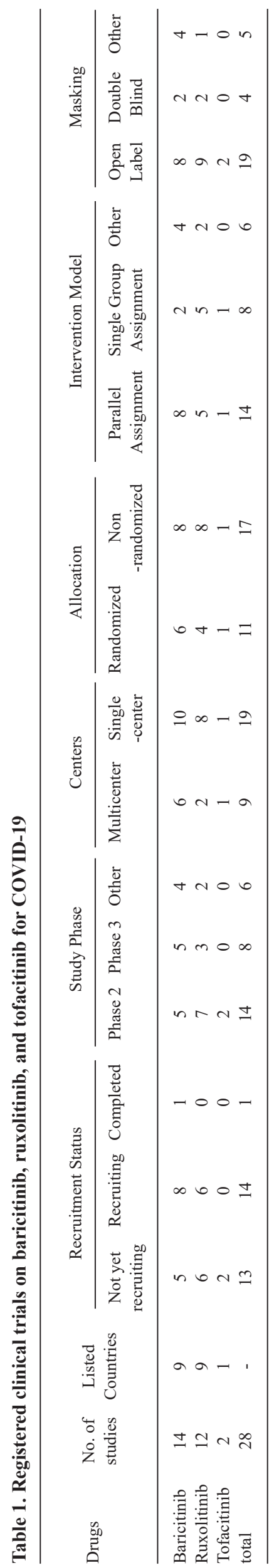


Conflict of Interest: The authors declare that they have no competing interests. This study was Approved by the ethics committee of Shanghai Public Health Clinical Center (Ethics number YJ-2020-S024-01).

\section{References}

1. World Health Organization. Public Health Emergency of International Concern declared. https://www.who.int/ emergencies/diseases/novel-coronavirus-2019/events-asthey-happen (accessed February 26, 2020).

2. Lai CC, Shih TP, Ko WC, Tang HJ, Hsueh PR. Severe acute respiratory syndrome coronavirus 2 (SARSCoV-2) and coronavirus disease-2019 (COVID-19): The epidemic and the challenges. Int $\mathrm{J}$ Antimicrob Agents. 2020; 55:105924.

3. Yang X, Yu Y, Xu J, et al. Clinical course and outcomes of critically ill patients with SARS-CoV-2 pneumonia in Wuhan, China: A single-centered, retrospective, observational study. Lancet Respir. Med. 2020; 8:475481.

4. Guan W-j, Ni Z-y, Hu Y, et al. Clinical characteristics of 2019 novel coronavirus infection in China. medRxiv. doi: https://doi.org/10.1101/2020.02.06.20020974

5. Wan S, Yi Q, Fan S, et al. Characteristics of lymphocyte subsets and cytokines in peripheral blood of 123 hospitalized patients with 2019 novel coronavirus pneumonia (NCP). medRxiv. 2020; https://doi. org/10.1101/2020.02.10.20021832

6. Mehta P, McAuley DF, Brown M, Sanchez E, Tattersall RS, Manson JJ. COVID-19: consider cytokine storm syndromes and immunosuppression. Lancet. 2020; 395:1033-1034.

7. Chen C, Zhang X, Ju Z, He W. Advances in the study of the mechanism of cytokine storms induced by COVID-19 and corresponding immunotherapies. Zhonghua Shao Shang Za Zhi. 2020; 36:E005. (in Chinese)

8. General Office of the National Health and Health Commission. Plan for Diagnosis and Treatment of Novel Coronavirus-related Pneumonia (Draft Version 6). http:// www.nhc.gov.cn/yzygj/s7653p/202003/46c9294a7dfe4cef 80dc7f5912eb1989/files/ce3e6945832a438eaae415350a8 ce964.pdf (accessed March 3, 2020). (in Chinese)

9. Xu X, Han M, Li T, Sun W, Wang D, Fu B, Zhou Y, Zheng X, Yang Y, Li X, Zhang X, Pan A, Wei H. Effective treatment of severe COVID-19 patients with tocilizumab. Proc Natl Acad Sci U S A. 2020; 117:1097010975

10. Richardson P, Griffin I, Tucker C, Smith D, Oechsle O, Phelan A, Stebbing J. Baricitinib as potential treatment for 2019-nCoV acute respiratory disease. Lancet. 2020; 395:e30-e31.

11. Cao Y, Wei J, Zou L, et al. Ruxolitinib in treatment of severe coronavirus disease 2019 (COVID-19): A multicenter, single-blind, randomized controlled trial. J Allergy Clin Immunol. 2020; S0091-6749(20)30738-7. doi: 10.1016/j.jaci.2020.05.019.

12. Xu X, Chen P, Wang J, Feng J, Zhou H, Li X, Zhong W, Hao P. Evolution of the novel coronavirus from the ongoing Wuhan outbreak and modeling of its spike protein for risk of human transmission. Sci China Life Sci. 2020; 63:457-460.

13. Zhao Y, Zhao Z, Wang Y, Zhou Y, Ma Y, Zuo W. Single-cell RNA expression profiling of ACE2, the putative receptor of Wuhan 2019-nCov. bioRxiv. 2020; doi: https://doi.org/10.1101/2020.01.26.919985.

14. Channappanavar R, Perlman S. Pathogenic human coronavirus infections: Causes and consequences of cytokine storm and immunopathology. Semin Immunopathol. 2017; 39:529-539.

15. Hui DSC, Zumla A. Severe acute respiratory syndrome: Historical, epidemiologic, and clinical features. Infect Dis Clin North Am. 2019; 33:869-889.

16. Reichsoellner M, Raggam RB, Wagner J, Krause R, Hoenigl M. Clinical evaluation of multiple inflammation biomarkers for diagnosis and prognosis for patients with systemic inflammatory response syndrome. J Clin Microbiol. 2014; 52:4063-4066.

17. Al-Jasser FS, Nouh RM, Youssef RM. Epidemiology and predictors of survival of MERS-CoV infections in Riyadh region, 2014-2015. J Infect Public Health. 2019; 12:171177.

18. Zhang B, Swamy S, Balijepalli S, Panicker S, Mooliyil J, Sherman MA, Parkkinen J, Raghavendran K, Suresh MV. Direct pulmonary delivery of solubilized curcumin reduces severity of lethal pneumonia. FASEB J. 2019; 33:13294-13309.

19. Krischuns T, Gunl F, Henschel L, Binder M, Willemsen J, Schloer S, Rescher U, Gerlt V, Zimmer G, Nordhoff C, Ludwig S, Brunotte L. Phosphorylation of TRIM28 enhances the expression of IFN-beta and proinflammatory cytokines during HPAIV infection of human lung epithelial cells. Front Immunol. 2018; 9:2229.

20. Kuldanek SA, Kelher M, Silliman CC. Risk factors, management and prevention of transfusion-related acute lung injury: A comprehensive update. Expert Rev Hematol. 2019; 12:773-785.

21. Tanaka T, Narazaki M, Masuda K, Kishimoto T. Regulation of IL-6 in immunity and diseases. Adv Exp Med Biol. 2016; 941:79-88.

22. Huang C, Wang Y, Li X, et al. Clinical features of patients infected with 2019 novel coronavirus in Wuhan, China. Lancet. 2020; 395:497-506.

23. Wu JH, Li X, Huang B, Su H, Li Y, Luo DJ, Chen S, Ma L, Wang SH, Nie X, Peng L. Pathological Changes of Fatal Coronavirus Disease 2019 (COVID-19) in the Lungs: Report of 10 Cases by Postmortem Needle Autopsy. Zhonghua Bing Li Xue Za Zhi. 2020; 6:568-575. (in Chinese)

24. $\mathrm{Xu} \mathrm{Z}$, Shi L, Wang Y, et al. Pathological findings of COVID-19 associated with acute respiratory distress syndrome. Lancet Respir Med. 2020; 8:420-422.

25. General Office of the National Health and Health Commission. Diagnosis and treatment of pneumonia caused by a novel coronavirus (Draft version 5). http:// www.gov.cn/zhengce/zhengceku/2020-02/05/5474791/ files/de44557832ad4be1929091dcbcfca891.pdf (accessed February 7, 2020). (in Chinese)

26. Shang L, Zhao J, Hu Y, Du R, Cao B. On the use of corticosteroids for 2019-nCoV pneumonia. Lancet. 2020; 395:683-684.

27. Faguer S, Vergez F, Peres M, Ferrandiz I, Casemayou A, Belliere J, Cointault O, Lavayssiere L, Nogier MB, Prevot G, Huart A, Recher C, Rostaing L. Tocilizumab added to conventional therapy reverses both the cytokine profile and $\mathrm{CD}^{+} \mathrm{Granzyme}^{+} \mathrm{T}$-cells/NK cells expansion in refractory hemophagocytic lymphohistiocytosis. Hematol Oncol. 2016; 34:55-57.

28. Cellina M, Orsi M, Bombaci F, Sala M, Marino P, Oliva 
G. Favorable changes of CT findings in a patient with COVID-19 pneumonia after treatment with tocilizumab. Diagn. Interv. Imaging. 2020; 101:323-324.

29. Gao J, Tian Z, Yang X. Breakthrough: Chloroquine phosphate has shown apparent efficacy in treatment of COVID-19 associated pneumonia in clinical studies. Biosci Trends. 2020; 14:72-73.

30. Guastalegname M, Vallone A. Could chloroquine/ hydroxychloroquine be harmful in coronavirus disease 2019 (COVID-19) treatment? Clin Infect Dis. 2020; ciaa321. doi: 10.1093/cid/ciaa321.

31. Shanghai Expert Group on Clinical Treatment of Novel Coronavirus Disease. Comprehensive treatment and management of corona virus disease 2019: Expert consensus statement from Shanghai. Chinese Journal of Infectious Diseases. 2020; 38. doi: 10.3760/cma. j.issn.1000- 6680.2020.0016.

32. Ning HB, Li K, Peng Z, Wang YG, Gu Y, Shang J. Continuous renal replacement therapy for a severe case of novel coronavirus-related pneumonia. Chinese Journal of Infectious Diseases. 2020; 38. doi: 10.3760/cma. j.cn311365-20200218-00079. (in Chinese)

33. Chinese Medical Association Expert Group on Kidney Disease. Recommendations for prevention and control of novel coronavirus infection in pediatric blood purification center. Chinese Journal of Nephrology, .2020; 36:82-84. (in Chinese)

34. Schwartz DM, Bonelli M, Gadina M, O'Shea JJ. Type I/II cytokines, JAKs, and new strategies for treating autoimmune diseases. Nat Rev Rheumatol. 2015; 12:2536.

35. Banerjee S, Biehl A, Gadina M, Hasni S, Schwartz DM. JAK-STAT signaling as a target for inflammatory and autoimmune disease: Current and future prospects. Drugs. 2017; 77:521-546.

36. Roskoski, Robert. Janus kinase (JAK) inhibitors in the treatment of inflammatory and neoplastic diseases. Pharmacol Res.2016; 111:784-803.

37. Schwartz DM, Kanno Y, Villarino A, Ward M, Gadina M, O'Shea JJ. JAK inhibition as a therapeutic strategy for immune and inflammatory diseases. Nat Rev Drug Discov.2017;16:843-862.

38. Chien JY, Hsueh PR, Cheng WC, Yu CJ, Yang PC. Temporal changes in cytokine/chemokine profiles and pulmonary involvement in severe acute respiratory syndrome. Respirology. 2006; 11:715-722.

39. Kong SL, Chui P, Lim B, Salto-Tellez M. Elucidating the molecular physiopathology of acute respiratory distress syndrome in severe acute respiratory syndrome patients. Virus Res. 2009; 145:260-269.

40. Cantini F, Niccoli L, Matarrese D, Nicastri E, Stobbione P, Goletti D. Baricitinib therapy in COVID-19: A pilot study on safety and clinical impact. J. Infect. 2020.

41. Stebbing J, Phelan A, Griffin I, Tucker C, Oechsle O, Smith D, Richardson P. COVID-19: Combining antiviral and anti-inflammatory treatments. Lancet Infect. Dis. 2020; $20: 400-402$.

42. Lurie N, Saville M, Hatchett R, Halton J. Developing Covid-19 vaccines at pandemic speed. N Engl J Med. 2020; 382:1969-1973.

Received April 22, 2020; Revised June 2, 2020; Accepted June 9, 2020.

*Address correspondence to:

Hongzhou Lu, Department of Infection and Immunity, Shanghai Public Health Clinical Center, Fudan University, Shanghai 201508, China.

E-mail: luhongzhou@fudan.edu.cn

Released online in J-STAGE as advance publication June 12, 2020. 